九州大学学術情報リポジトリ

Kyushu University Institutional Repository

\title{
STOPPING RULES FOR SEQUENTIAL DENSITY ESTIMATION
}

\section{Isogai, Ei ichi}

Department of Mathematics, Faculty of Science, Niigata University

https://doi.org/10.5109/13148

出版情報: 統計数理研究. 19 (3/4)，pp.53-67，1981-03. Research Association of Statistical Sciences バージョン :

権利関係 : 


\title{
STOPPING RULES FOR SEQUENTIAL DENSITY ESTIMATION
}

\author{
By
}

\author{
Eiichi IsoGAI*
}

(Received October 30, 1980)

\section{Introduction.}

There have been several papers on the sequential estimation of a probability density function (p.d.f.) $f(x)$ and stopping rules. Yamato [10], Wegman and Davies [9] and Isogai [6] proposed recursive density estimators. On the other hand, Davies and Wegman [4] treated the problem of sequential stopping rules. Carroll [2] developed two classes of stopping rules for estimating $f(x)$, one of which yields a confidence interval for $f(x)$ of fixed-width and prescribed coverage probability.

In this paper we consider the problem of estimating $f(x)$ at a given point $x$, where $f(x)$ is a (unknown) p.d.f. on the $p$-dimensional Euclidean space $R^{p}$ with respect to Lebesgue measure. We then use the recursive estimator $f_{n}(x)$ proposed by Isogai [6] and give a class of stopping rules for estimating $f(x)$ which is similar to that of Carroll [2]. One of the differences between our paper and Carroll's [2] is that the recursive density estimators which appear in specifications of stopping rules are different.

In Section 2 we shall present the recursive density estimator $f_{n}(x)$ and auxiliary results obtained by Isogai [6] needed for Section 3. In Section 3 we shall give a class of stopping rules for estimating $f(x)$ and examine the asymptotic behaviors of the rules; the stopping rules yield a confidence interval for $f(x)$ of fixed-width $2 d$ and prescribed coverage probability $1-\alpha$. This asymptotic behavior can be obtained by the use of the asymptotic normality of $f_{n}(x)$ and the verification of Anscombe's condition. Moreover we shall discuss the asymptotic normality of the stopping rules.

\section{Preliminaries.}

In this section we shall make preparations for Section 3. Let $K(y)$ be a real-valued Borel function on $R^{p}$ satisfying

(K1) $K(y) \geqq 0$ on $R^{p}$ and $\int K(y) d y=1$,

* Department of Mathematics, Faculty of Science, Niigata University, Niigata, Japan. 
(K2) $\|K\|_{\infty}=\sup _{y \in R^{p}} K(y)<\infty$,

(K3) $\lim _{y=\infty \rightarrow \infty}\|y\|_{i}^{p} K(y)=0$

and

(K4) $\int\|y\| K(y) d y<\infty$,

where $\|\cdot\|$ denotes the Euclidean norm and the domain of integral is $R^{p}$ unless otherwise specified. Let $\left\{a_{n}\right\}$ be a sequence of positive numbers defined by

$$
a_{n}=\frac{a}{n} \quad \text { with } \quad \frac{1}{2}<a \leqq 1 \quad \text { for all } n \geqq 1 .
$$

Let $\left\{h_{n}\right\}$ be a sequence of positive numbers. In Section 3, on this sequence we shall impose some or all of the following conditions:

(H1) $\lim _{n \rightarrow \infty} h_{n}=0$,

(H2) $\quad h_{n_{0}} \geqq h_{n_{0}+1} \geqq \cdots \quad$ for some $\quad n_{0} \geqq 1$,

(H3) $\lim _{n \rightarrow \infty} n h_{n}^{p}=\infty$,

(H4) $n_{0} h_{n_{0}}^{p} \leqq\left(n_{0}+1\right) h_{n_{0}+1}^{p} \leqq \cdots \quad$ for some $\quad n_{0} \geqq 1$,

(H5) $\lim _{n \rightarrow \infty} \frac{h_{n}}{h_{n+1}}=1$,

(H6) $\lim _{n \rightarrow \infty}\left(n h_{n}^{p}\right)^{-1 / 2} \log n=0$,

(H7) $\sum_{n=1}^{\infty}\left(n^{2} h_{n}^{p}\right)^{-1}<\infty$.

We should note that (H6) implies (H3). Define $K_{n}(x, y)$ by

$$
K_{n}(x, y)=h_{n}^{-p} K\left(\frac{x-y}{h_{n}}\right) \quad \text { for all } \quad x, y \in R^{p}, n=1,2, \cdots .
$$

The following recursive density estimator is proposed by the author [6].

$$
\begin{array}{ll}
f_{0}(x)=K(x) & \text { for all } x \in R^{p} \\
f_{n}(x)=\left(1-a_{n}\right) f_{n-1}(x)+a_{n} K_{n}\left(x, X_{n}\right) & \text { for all } x \in R^{p}, n=1,2, \cdots,
\end{array}
$$

where $X_{1}, X_{2}, X_{3}, \cdots$ is a sequence of independent identically distributed $p$-dimensional random vectors with the common (unknown) p.d.f. $f$ on some probability space $(\Omega, \mathscr{B}, P)$, and the conditions $(\mathrm{K} 1) \sim(\mathrm{K} 4),(\mathrm{A})$ and $(\mathrm{H} 1)$ are assumed to be satisfied. In what follows, for the estimator $f_{n}$ we shall assume the conditions (K1) (K4), (A) and (H1) without restating them repeatedly. Throughout this paper $C_{1}, C_{2}, \cdots$ denote positive constants, and for any function $g C(g)$ stands for the set of all points of continuity of $g$.

REMARK. It is easy to see that $f_{n}(x)(n=1,2, \cdots)$ are probability density functions. We shall, now, introduce some notations. Let 


$$
\begin{aligned}
\beta_{m n} & =\prod_{k=m+1}^{n}\left(1-a_{k}\right) \quad \text { if } \quad 0 \leqq m<n \\
& =1 \quad \text { if } \quad m=n \geqq 0 \\
\gamma_{0}=\gamma_{1}=1 &
\end{aligned}
$$

and

$$
\gamma_{n}=\prod_{j=2}^{n}\left(1-a_{j}\right) \quad \text { for } \quad n \geqq 2 .
$$

It is clear that $\gamma_{n}>0$ for all $n \geqq 1$ and $\beta_{m n}=\gamma_{n} \gamma_{m}^{-1}$ for all $n \geqq m \geqq 1$. The author [6] gave the inequalities:

$$
C_{1} m^{a} n^{-a} \leqq \beta_{m n} \leqq C_{2} m^{a} n^{-a} \quad \text { for all } n \geqq m \geqq 1 .
$$

Definition 2.1. A bounded real-valued function $g$ defined on $R^{p}$ is said to be locally Lipschitz of order $\lambda, 0<\lambda \leqq 1$, at $x_{0}$ (abbreviated as loc. Lip. $\lambda$ at $x_{0}$ ) if there exist two positive constants $L$ and $\eta$, depending on $x_{0}$ and $\lambda$ such that $\|y\|<\eta$ implies $\left|g\left(x_{0} \div y\right)-g\left(x_{0}\right)\right| \leqq L\|y\|^{\lambda}$.

The following lemma was given by the author [6].

LEMma 2.2. Let $Z_{n}=K_{n}\left(x, X_{n}\right)-E K_{n}\left(x, X_{n}\right)$ for all $n \geqq 1$. Assume that $\left\{h_{n}\right\}$ satisfies (H1), (H2) and (H6). In addition suppose the following conditions:

(2.2.1) For some $a$ in (A) with $\frac{2}{3} \leqq a \leqq 1$ there exists a positive constant $\beta$ such that

$$
n^{1-2 a} h_{n}^{p} \sum_{m=1}^{n} m^{2(a-1)} h_{m}^{-p} \longrightarrow \beta \quad \text { as } n \rightarrow \infty,
$$

$$
\|f\|_{\infty}=\sup _{y \in R^{p}} f(y)<\infty .
$$

Then, for each $x \in C(f)$

$$
\left(n h_{n}^{p}\right)^{1 / 2} \sum_{m=1}^{n} a_{m} \beta_{m n} Z_{m} \underset{\mathcal{L}}{\longrightarrow} N(0, B f(x)) \quad \text { as } n \rightarrow \infty,
$$

where $B=a^{2} \beta \int K^{2}(y) d y, N\left(0, \sigma^{2}\right)$ stands for the normal random variable with mean 0 and variance $\sigma^{2}$ and " $\underset{\mathcal{L}}{\longrightarrow}$ means convergence in law.

\section{Stopping rules.}

In this section we shall propose a class of stopping rules which yields a confidence interval for $f(x)$ of prescribed width $2 d$ and prescribed coverage probability $1-\alpha$. The asymptotic normality of the stopping rules will also be shown. Our stopping rules are analogous to those of Carroll [2], but in the specifications of the stopping rules the recursive estimators of Carroll [2] and Isogai [6] are different.

Let any $\alpha(0<\alpha<1)$ be given. Define $D=D(\alpha)$ by $D(\alpha)=\Phi^{-1}\left(1-\frac{\alpha}{2}\right)$, where $\Phi$ is 
the distribution function of the standard normal random variable. We shall, now, define stopping rules as follows:

$$
N(d)=\text { the smallest integer } n \geqq 1 \text { such that }
$$

$$
\left(D^{2} B\right)^{-1} n h_{n}^{p} d^{2} \geqq f_{n}(x)>0,
$$

where $B$ is the same as in Lemma 2.2 and $f_{n}(x)$ is defined by $(\mathrm{F})$. Also, define $n(d)$ by

$$
\begin{aligned}
& n(d)=\text { the smallest integer } n \geqq 1 \text { such that } \\
& \qquad\left(D^{2} B\right)^{-1} n h_{n}^{p} d^{2} \geqq f(x) .
\end{aligned}
$$

The following lemma is a modification of Lemma 1 in Chow and Robbins [3].

LEMMA 3.1. Let $y_{n}(n=1,2, \cdots)$ be any sequence of random variables on a probability space $(\Omega, \mathscr{I}, P)$ satisfying the following: There exists a null set $\tilde{N}_{1}\left(i . e . P\left\{\tilde{N}_{1}\right\}=0\right)$ such that for each $\omega \in \tilde{N}_{1}^{c}$, where $\tilde{N}_{1}^{c}$ is the complement of the set $\tilde{N}_{1}$,

$$
y_{n}(\omega) \geqq 0 \quad \text { for all } n \geqq 1 \text { and } \lim _{n \rightarrow \infty} y_{n}(\omega)=1 \text {, }
$$

and if $y_{m}(\omega)>0$ for some $m=m(\omega)$ then $y_{n}(\omega)>0$ for all $n \geqq m$. Let $\{g(n)\}$ be any sequence of constants such that

$$
g(n)>0, \quad \lim _{n \rightarrow \infty} g(n)=\infty \quad \text { and } \quad \lim _{n \rightarrow \infty} \frac{g(n)}{g(n-1)}=1,
$$

and for each $t>0$ and any fixed integer $n_{1} \geqq 1$ define

$$
N_{t}=N\left(t, n_{1}\right)=\text { the smallest integer } n \geqq n_{1} \text { such that }
$$

$$
0<y_{n} \leqq \frac{g(n)}{t} .
$$

Then, $N_{t}$ is well-defined and non-decreasing as a function of $t$,

$$
\lim _{t \rightarrow \infty} N_{t}=\infty \quad \text { a.s. }
$$

and

$$
\lim _{t \rightarrow \infty} \frac{g\left(N_{t}\right)}{t}=1 \text { a.s. }
$$

Proof. It is easy to see that $N_{t}$ is well-defined and non-decreasing as a function of $t$. Hence there exists $\lim _{t \rightarrow \infty} N_{t}$ on $\tilde{N}_{1}^{c}$. First we shall show (3.1.1). Suppose that there exists a set $\Omega^{1} \in \mathscr{F}$ with $P\left\{\Omega^{1}\right\}>0$ such that

$$
M=\lim _{t \rightarrow \infty} N_{t}<\infty \quad \text { on } \quad \Omega^{1}\left(\subset \tilde{N}_{1}^{c}\right) .
$$

Let any $\omega \in \Omega^{1}$ be fixed. By definition of $N_{t}$ and (3.1.3) we get

$$
y_{M}=\lim _{t \rightarrow \infty} y_{N_{t}} \leqq \lim _{t \rightarrow \infty} \frac{g\left(N_{t}\right)}{t}=0,
$$


where we omit $\omega$. Thus we have

$$
y_{M}=0 \text {. }
$$

Let any $t>0$ be fixed. By the monotonicity of $N_{t}$ we get $M \geqq N_{t}$. Taking account of $y_{N_{t}}>0$ and assumption of $y_{n}$, we have $y_{M}>0$, which contradicts (3.1.4). Thus we have $P\left\{\Omega^{1}\right\}=0$, concluding (3.1.1). Finally we shall show (3.1.2). Let $\tilde{N}_{2}=\tilde{N}_{1}^{c},\{\omega \in \Omega$ : $\left.\lim _{t \rightarrow \infty} N_{t}=\infty\right\}$. It follows from the above relation that $P\left\{\tilde{N}_{2}^{c}\right\}=1$. Let any $\omega \in \tilde{N}_{2}^{c}$ be fixed. For convenience we omit $\omega$. As $\lim _{n \rightarrow \infty} y_{n}=1$, there exists a positive integer $n_{2}>n_{1}$ such that

$$
y_{n}>0 \quad \text { for all } n \geqq n_{2} .
$$

On the other hand, as $\lim _{t \rightarrow \infty} N_{t}=\infty$, there exists a positive number $t_{0}$ such that

$$
N_{t}-1 \geqq n_{2} \quad \text { for all } t \geqq t_{0},
$$

which, together with (3.1.5), yields

$$
y_{v_{t^{-1}}}>0 \text { for all } t \geqq t_{0} .
$$

Hence by (3.1.6) and definition of $N_{t}$ we get

$$
y_{N_{t}} \leqq \frac{g\left(N_{t}\right)}{t}<\frac{g\left(N_{t}\right)}{g\left(N_{t}-1\right)} y_{N_{t^{-1}}} \quad \text { for all } t \geqq t_{0} .
$$

Since $\lim _{t \rightarrow \infty} \frac{g\left(N_{t}\right)}{g\left(N_{t}-1\right)}=1$, it follows from (3.1.7) and assumption of $y_{n}$ that $\lim _{t \rightarrow \infty} \frac{g\left(N_{t}\right)}{t}$ $=1$ on $\tilde{N}_{2}^{c}$, concluding (3.1.2). Thus the proof is complete.

Theorem 3.2. Suppose that $\left\{h_{n}\right\}$ satisfies (H1), (H3), (H5) and (H7). Then, for each point $x \in C(f)$ with $f(x)>0, N(d)$ is well-defined. Furthermore,

$$
\lim _{d i 0} N(d)=\infty \quad \text { a.s. }
$$

and

$$
\lim _{d \vee 0} \frac{N(d) h_{v(d)}^{p} d^{2}}{D^{2} B f(x)}=1 \text { a.s. }
$$

Proof. By (3.0) we get

$$
0<\frac{f_{n}(x)}{f(x)} \leqq \frac{n h_{n}^{p}}{f(x)} /\left(D^{2} B d^{-2}\right) .
$$

In Lemma 3.1 we set

$$
y_{n}=\frac{f_{n}(x)}{f(x)}, \quad g(n)=\frac{n h_{n}^{p}}{f(x)} \quad \text { and } \quad t=D^{2} B d^{-2} \quad \text { for all } n \geqq 1 .
$$

By Theorem 3.1 of Isogai [6] there exists a null set $\tilde{N}_{1} \in \mathscr{B}$ such that $\lim _{n \rightarrow \infty} y_{n}=1$ on $\tilde{N}_{1}^{c}$. By Remark in Section 2 it follows that

$$
y_{n} \geqq 0 \quad \text { on } \quad \Omega \quad \text { for all } n \geqq 1 .
$$

All conditions of $\{g(n)\}$ in Lemma 3.1 are satisfied by (H3) and (H5). Suppose that 
for any fixed $\omega \subseteq \tilde{N}_{1}^{c}$ omitted below there exists a positive integer $m$ such that $y_{m}>0$. If $y_{n}>0$ for $n>m$ which is equivalent to $f_{n}(x)>0$, according to $K_{n+1}\left(x, X_{n+1}\right) \geqq 0$ and $1-a_{n+1}>0$ we have $f_{n+1}(x)>0$ which is equivalent to $y_{n+1}>0$. Thus by induction we get $y_{n}>0$ for all $n \geqq m$. Since all conditions of Lemma 3.1 are satisfied, Lemma 3.1 yields (3.2.1) and (3.2.2). The proof is complete.

By the same argument for Theorem 3.2 it follows that under (H1), (H3), (H5) and $f(x)$ $>0 n(d)$ is non-decreasing as a function of $d$, and that

$$
\lim _{d \downarrow 0} n(d)=\infty
$$

and

$$
\lim _{d \downarrow 0} \frac{n(d) h_{n(d)}^{p} d^{2}}{D^{2} B f(x)}=1
$$

Thus by (3.2.2) and (3.2.4) we obtain

COROLLARY 3.3. Under all conditions of Theorem 3.2 we have

$$
\lim _{d \downarrow 0} \frac{N(d) h_{N(d)}^{p}}{n(d) h_{n(d)}^{p}}=1 \quad \text { a.s. }
$$

By Theorem 3.1 of Isogai [6], Theorem 3.2 and Theorem 1 of Richter [8] we have Proposition 3.4. Under all conditions of Theorem 3.2 we have

$$
\lim _{d \downarrow 0} f_{N\langle d\rangle}(x)=f(x) \text { a.s. }
$$

Now, we shall verify Anscombe's condition.

LEMMA 3.5. Suppose that $\left\{h_{n}\right\}$ satisfies (H1), (H2), (H4) and (2.2.1). Let any $x \in C(f)$ with $f(x)>0$ be fixed and set $S_{n}=f_{n}(x)-E f_{n}(x)$. Then, for any positive numbers $\varepsilon$ and $\eta$, there exist a positive constant $\rho=\rho(\varepsilon, \eta)<1$ and a positive integer $\nu=\nu(\varepsilon, \eta)$ such that

$$
P\left\{\max _{\mid i-n ! \geqq n \rho}\left|S_{i}-S_{n}\right| \geqq \varepsilon\left(n h_{n}^{p}\right)^{-1 / 2}\right\}<\eta \quad \text { for all } n \geqq \nu .
$$

Proof. Let $\varepsilon_{n}=\varepsilon\left(n h_{n}^{p}\right)^{-1 / 2}$. In what follows we consider $\rho$ with $0<\rho \leqq \frac{1}{2}$ and $n$ satisfying $n \rho>1$ for fixed $\rho$ and $n \geqq 2 n_{0}$ which is the same as in (H2) and (H4). For any fixed $\rho$ and $n$ define two positive integers $m_{1}=m_{1}(\rho, n)$ and $m_{2}=m_{2}(\rho, n)$ by

$$
(1-\rho) n \leqq m_{1}<(1-\rho) n+1 \text { and }(1+\rho) n-1<m_{2} \leqq(1+\rho) n \text {. }
$$

We note that $m_{2}>m_{1} \geqq n_{0}$ and $m_{2}>n>m_{1}$ for each $\rho$ and $n$. Since $S_{n}$ can be rewritten as $\sum_{m=1}^{n} a_{m} \beta_{m n} Z_{m}$ with $Z_{m}$ being the same as in Lemma 2.2, we have

$$
\begin{aligned}
& P\left\{\max _{: i-n \mid \geqq n \rho}\left|S_{i}-S_{n}\right| \geqq \varepsilon_{n}\right\} \\
& \quad \leqq P\left\{\max _{m_{1} \leq i \leqq n-1}\left|S_{i}-S_{n}\right| \geqq \varepsilon_{n}\right\}+P\left\{\max _{n+1 \leqq i \leqq m_{2}}\left|S_{i}-S_{n}\right| \geqq \varepsilon_{n}\right\}
\end{aligned}
$$




$$
\begin{aligned}
\leqq & P\left\{\max _{m_{1} \leqq i \leqq n-1}\left|\sum_{m=1}^{i} a_{m}\left(\beta_{m i}-\beta_{m n}\right) Z_{m}\right| \geqq \frac{\varepsilon_{n}}{2}\right\} \\
& +P\left\{\max _{m_{1} \leqq i \leqq n-1}\left|\sum_{m=i+1}^{n} a_{m} \beta_{m n} Z_{m}\right| \geqq \frac{\varepsilon_{n}}{2}\right\} \\
& +P\left\{\max _{n+1 \leqq i \leqq m_{2}}\left|\sum_{m=1}^{n} a_{m}\left(\beta_{m i}-\beta_{m n}\right) Z_{m}\right| \geqq \frac{\varepsilon_{n}}{2}\right\} \\
& +P\left\{\max _{n+1 \leqq i \leqq m_{2}}\left|\sum_{m=n+1}^{i} a_{m} \beta_{m i} Z_{m}\right| \geqq \frac{\varepsilon_{n}}{2}\right\} \\
= & I_{1}+I_{2}+I_{3}+I_{4}, \text { say. }
\end{aligned}
$$

Define

$$
\begin{aligned}
& d_{m m_{1}}=\beta_{m m_{1}} \quad \text { if } \quad m \leqq m_{1} \\
& =\beta_{m_{1} m}^{-1} \text { if } m>m_{1} \text {. }
\end{aligned}
$$

Then it is easy to see that $\beta_{m i}-\beta_{m n}=\left(1-\beta_{i n}\right) \beta_{m_{1} i} d_{m m_{1}}$ for $m_{1} \leqq i \leqq n-1$ and $m \leqq i$, and that $\left(1-\beta_{i n}\right) \beta_{m_{1} i}$ is positive and non-increasing as a function of $i$ for $m_{1} \leqq i \leqq n-1$. Hence by the Hájek-Rényi inequality (see Hájek and Rényi [5]) we get

$$
\begin{aligned}
I_{1}= & P\left\{\max _{m_{1} \leqq i \leqq n-1}\left(1-\beta_{i n}\right) \beta_{m_{1} i}\left|\sum_{m=1}^{i} a_{m} d_{m m_{1}} Z_{m}\right| \geqq \frac{\varepsilon_{n}}{2}\right\} \\
\leqq & \left(2 / \varepsilon_{n}\right)^{2}\left(1-\beta_{m_{1} n}\right)^{2} \beta_{m_{1} m_{1}}^{2} \sum_{m=1}^{m_{1}} a_{m}^{2} d_{m m_{1}}^{2} E Z_{m}^{2} \\
& \quad+\left(2 / \varepsilon_{n}\right)^{2} \sum_{m=m_{1}+1}^{n-1}\left(1-\beta_{m n}\right)^{2} \beta_{m_{1} m}^{2} a_{m}^{2} d_{m m_{1}}^{2} E Z_{m}^{2} \\
& =J_{1}+J_{2}, \text { say. }
\end{aligned}
$$

As $\operatorname{Var}\left(S_{n}\right)=\sum_{m=1}^{n} a_{m}^{2} \beta_{m n}^{2} E Z_{m}^{2}$, by Theorem 3.2 of Isogai [6] we get

$$
n h_{n}^{p} \sum_{m=1}^{n} a_{m}^{2} d_{m n}^{2} E Z_{m}^{2} \sim B f(x) \quad \text { as } \quad n \rightarrow \infty
$$

where " $\phi_{n} \sim \phi_{n}$ as $n \rightarrow \infty$ " means that $\phi_{n} / \phi_{n} \rightarrow 1$ as $n \rightarrow \infty$.

The relation (3.5.2) implies that

$$
m_{1} / n \sim 1-\rho \quad \text { as } n \rightarrow \infty .
$$

It follows from (H2) and (H4) that

$$
1 \leqq n h_{n}^{p} /\left(m_{1} h_{m_{1}}^{p}\right) \leqq n / m_{1}
$$

Let any $o_{1}$ with $0<\rho_{1} \leqq \frac{1}{2}$ be fixed. From (3.5.6) and (3.5.7) we get

$$
n h_{n}^{p} /\left(m_{1} h_{m_{1}}^{p}\right) \leqq 4 \text { for } n \text { sufficiently large }
$$

It follows from (3.5.5) that 


$$
n h_{n}^{p} \sum_{n=1}^{n} a_{m}^{2} d_{m n}^{2} E Z_{m}^{2} \leqq C_{3} \quad \text { for all } n \geqq 1
$$

Combining (3.5.8) and (3.5.9) we have

$$
J_{1} \leqq C_{4}(\varepsilon)\left(1-\beta_{m_{1} n}\right)^{2} \text { for } n \text { sufficiently large . }
$$

By making use of (3.5.6) and the fact that $\beta_{m n} \sim m^{a} n^{-a}$ as $m \rightarrow \infty$, we get $\left(1-\beta_{m_{1} n}\right)^{2}$ $\sim\left\{1-\left(1-\rho_{1}\right)^{a}\right\}^{2}$ as $n \rightarrow \infty$. Hence we have $\left(1-\beta_{m_{1} n}\right)^{2} \leqq 2\left\{1-\left(1-\rho_{1}\right)^{a}\right\}^{2}$ for $n$ sufficiently large, which, together with (3.5.10), implies that

$$
J_{1} \leqq C_{5}(\varepsilon)\left\{1-\left(1-\rho_{1}\right)^{a}\right\}^{2} \text { for } n \text { sufficiently large . }
$$

Choose $0<\rho_{1}=\rho_{1}(\varepsilon, \eta)<\frac{1}{2}$ such that $C_{5}(\varepsilon)\left\{1-(1-\rho)^{a}\right\}^{2}<\frac{\eta}{8}$ for all $0<\rho \leqq \rho_{1}$. Thus, for any fixed $\rho_{2}$ with $0<\rho_{2} \leqq \rho_{1}$, it follows from (3.5.11) that

$$
J_{1}<\eta / 8 \text { for } i \text { sufficiently large. }
$$

In the proof of Theorem 3.2 of Isogai [6] it has been shown that

$$
h_{n}^{p} E Z_{n}^{2} \leqq C_{6} \quad \text { for all } n \geqq 1 .
$$

In view of $(\mathrm{H} 2)$ and (3.5.13) we have

$$
\begin{aligned}
& J_{2} \leqq C_{i}(\varepsilon) n h_{n}^{p} \sum_{m=m_{1}+1}^{n-1} m^{-1} m^{-1} h_{m}^{-p} \\
& \leqq C_{7}(\varepsilon)\left(n / m_{1}\right) \sum_{m=m_{1+1}}^{n-1} m^{-1} \\
& \leqq C_{7}(\varepsilon)\left(n / m_{1}\right) \log \left(n / m_{1}\right) .
\end{aligned}
$$

Since by (3.5.6) $\left(n / m_{1}\right) \log \left(n / m_{1}\right) \sim\left(1-\rho_{2}\right)^{-1} \log \left(1-\rho_{2}\right)^{-1}$ as $n \rightarrow \infty$, it follows that

$$
\left(n / m_{1}\right) \log \left(n / m_{1}\right) \leqq 2\left(1-\rho_{2}\right)^{-1} \log \left(1-\rho_{2}\right)^{-1}
$$

for $n$ sufficiently large. Choose $0<\rho_{2}=\rho_{2}(\varepsilon, \eta) \leqq \rho_{1}$ such that

$$
C_{7}(\varepsilon)(1-\rho)^{-1} \log (1-\rho)^{-1}<\eta / 16 \quad \text { for all } 0<\rho \leqq \rho_{2} .
$$

Thus, for any fixed $\rho_{3}$ with $0<\rho_{3} \leqq \rho_{2}$, it follows from (3.5.14) and (3.5.15) that

$$
J_{2}<\eta / 8 \text { for } n \text { sufficiently large. }
$$

Combining (3.5.4), (3.5.12) and (3.5.16) we have the following:

For any fixed $\rho_{3}$ such that $0<\rho_{3} \leqq \rho_{2}$

$$
I_{1}<\eta / 4 \text { for } n \text { sufficiently large. }
$$

Now, let any $\rho_{3}$ with $0<\rho_{3} \leqq \rho_{2}$ be fixed. By Kolmogorov's inequality we get

$$
\begin{aligned}
I_{2} & \leqq(2 / \varepsilon)^{2}\left\{n h_{n}^{p} \sum_{m=1}^{n} a_{m}^{2} \beta_{m n}^{2} E Z_{m}^{2}-n h_{n}^{p} \sum_{m=1}^{m_{1}} a_{m}^{2} \beta_{m n}^{2} E Z_{m}^{2}\right\} \\
& \leqq(2 / \varepsilon)^{2} T(n)\left\{1-\beta_{m_{1} n}^{2}\left(n h_{n}^{p} / m_{1} h_{m_{1}}^{p}\right)\left(T\left(m_{1}\right) / T(n)\right)\right\}
\end{aligned}
$$


where $T(n)=n h_{n}^{p} \sum_{m=1}^{n} a_{m}^{2} \beta_{m n}^{2} E Z_{m}^{2}$. It follows from (3.5.9) that

$$
T(n) \leqq C_{3} \quad \text { for all } n \geqq 1 .
$$

Hence, in view of (3.5.7), (3.5.18) and (3.5.19) we have

$$
I_{2} \leqq C_{8}(\varepsilon)\left\{1-\beta_{m_{1} n}^{2}\left(T\left(m_{1}\right) / T(n)\right)\right\} \quad \text { for all } n .
$$

As it is easy to see that $1-\beta_{m_{1} n}^{2}\left(T\left(m_{1}\right) / T(n)\right) \leqq 2\left\{1-\left(1-\rho_{3}\right)^{2 a}\right\}$ for $n$ sufficiently large, by (3.5.20) and the discussion similar to (3.5.17) we can choose $0<\rho_{3}=\rho_{3}(\varepsilon, \eta) \leqq \rho_{2}$ such that for any fixed $\rho_{4}$ with $0<\rho_{4} \leqq \rho_{3}$

$$
I_{2}<\eta / 4 \text { for } n \text { sufficiently large. }
$$

Let any $\rho_{4}$ with $0<\rho_{4} \leqq \rho_{3}$ be fixed. By making use of (3.5.9) and Chebychev's inequality we have

$$
\begin{aligned}
I_{3} & =P\left\{\left(\gamma_{n}-\gamma_{m_{2}}\right)\left|\sum_{m=1}^{n} a_{m} \gamma_{m}^{-1} Z_{m}\right| \geqq \frac{\varepsilon_{n}}{2}\right\} \\
& \leqq(2 / \varepsilon)^{2}\left(1-\beta_{n m_{2}}\right)^{2} n h_{n}^{p} \sum_{m=1}^{n} a_{m}^{2} \beta_{m n}^{2} E Z_{m}^{2} \\
& \leqq C_{9}(\varepsilon)\left(1-\beta_{n m_{2}}\right)^{2} .
\end{aligned}
$$

As

$$
m_{2} / n \sim 1+\rho_{4} \quad \text { as } \quad n \rightarrow \infty,
$$

we get $\left(1-\beta_{n m_{2}}\right)^{2} \leqq 2\left\{1-\left(1+\rho_{4}\right)^{-a}\right\}^{2}$ for $n$ sufficiently large. Thus, using (3.5.22) we can choose $0<\rho_{4}=\rho_{4}(\varepsilon, \eta) \leqq \rho_{3}$ such that for any fixed $\rho$ with $0<\rho \leqq \rho_{4}$

$I_{3}<\eta / 4$ for $n$ sufficiently large.

Finally, let any $\rho$ with $0<\rho \leqq \rho_{4}$ be fixed. Using (H4), (3.5.13) and the Hájek-Rényi inequality, we have

$$
\begin{aligned}
I_{4} & =P\left\{\max _{n+1 \leqq i \leqq m_{2}} \gamma_{i}\left|\sum_{m=n+1}^{i} a_{m} \gamma_{m}^{-1} Z_{m}\right| \geqq \frac{\varepsilon_{n}}{2}\right\} \\
& \leqq(2 / \varepsilon)^{2} n h_{n}^{p} \sum_{m=n+1}^{m_{2}} a_{m}^{2} E Z_{m}^{2} \\
& \leqq C_{10}(\varepsilon) \sum_{m=n+1}^{m_{2}} m^{-1} \\
& \leqq C_{10}(\varepsilon) \log \left(m_{2} / n\right) .
\end{aligned}
$$

As by $(3.5 .23) \log \left(m_{2} / n\right) \sim \log (1+\rho)$ as $n \rightarrow \infty$, we get $\log \left(m_{2} / n\right) \leqq 2 \log (1+\rho)$ for $n$ sufficiently large. Thus, using (3.5.25) we can choose $0<\rho=\rho(\varepsilon, \eta) \leqq \rho_{4}$ such that for $\rho$

$$
I_{4}<\eta / 4 \text { for } n \text { sufficiently large. }
$$

For $\rho$ chosen above, choose a positive integer $\nu$ such that for all $n \geqq \nu$ (3.5.17), (3.5.21), (3.5.24) and (3.5.26) hold. Thus, combining (3.5.3), (3.5.17), (3.5.21), (3.5.24) and (3.5.26) 
we obtain (3.5.1). This completes the proof.

The following theorem is concerned with the asymptotic normality of $f_{x(d)}(x)$.

THEOREM 3.6. Assume (2.2.1). Suppose that $f$ is loc. Lip. $\lambda$ at $x$ and $f(x)>0$, and that $\left\{h_{n}\right\}$ satisfies $(\mathrm{H} 1) \sim(\mathrm{H} 7)$ except for $(\mathrm{H} 3)$. Furthermore suppose the following conditions:

$$
\sum_{m=1}^{n} m^{a-1} h_{m}^{\lambda}=O\left(n^{a} h_{n}^{\lambda}\right) \quad \text { as } n \rightarrow \infty
$$

with a being the same as in (2.2.1),

$$
\begin{aligned}
& \lim _{n \rightarrow \infty} n h_{n}^{2 \lambda+p}=0, \\
& \lim _{d \downarrow 0} N(d) / n(d)=1 \text { in probability. }
\end{aligned}
$$

Then we have

$$
\left(N(d) h_{Y(d)}^{p}\right)^{1 / 2}\left(f_{N(d)}(x)-f(x)\right) \underset{\mathcal{L}}{\longrightarrow} N(0, B f(x)) \quad \text { as } \quad d \downarrow 0,
$$

where $B$ is the same as in Lemma 2.2 .

Proof. Since, by $(F)$ in Section 2,

$$
\begin{aligned}
\left(n h h_{n}^{p}\right)^{1 / 2} & \left(f_{n}(x)-f(x)\right) \\
= & \left(n h_{n}^{p}\right)^{1 / 2} \sum_{m=1}^{n} a_{m} \beta_{m n} Z_{m}+\left(n h_{n}^{p}\right)^{1 / 2} \sum_{m=1}^{n} a_{m} \beta_{m n} \delta_{m} \\
& +\left(n h_{n}^{p}\right)^{1 / 2} \beta_{0 n}\left(f_{0}(x)-f(x)\right),
\end{aligned}
$$

where $\delta_{m}=E K_{m}\left(x, X_{m}\right)-f(x)$ and $Z_{m}$ is the same as in Lemma 2.2, we have (3.6.4) if it holds that as $d \downarrow 0$

$$
\begin{aligned}
& \left(N(d) h_{N(d)}^{p}\right)^{1 / 2} \sum_{m=1}^{N(d)} a_{m} \beta_{m N(d)} Z_{m} \underset{\mathcal{L}}{\longrightarrow} N(0, B f(x)), \\
& \left(N(d) h_{:(d)}^{p}\right)^{1 / 2} \sum_{m=1}^{N(d)} a_{m} \beta_{m N(d)} \delta_{m} \longrightarrow 0 \quad \text { in probability }
\end{aligned}
$$

and

$$
\left(N(d) h_{N(d)}^{p}\right)^{1 / 2} \beta_{0 N(d)}\left(f_{0}(x)-f(x)\right) \longrightarrow 0 \text { in probability. }
$$

First we shall show (3.6.6). For convenience $N$ denotes $N(d)(\omega)$ for each $\omega \subseteq \Omega$ and $d$. Let any $d_{0}>0$ be fixed. Setting $T=\left\{\omega \in \Omega: N(d)(\omega)<\infty\right.$ for all $0<d<d_{0}$ and $\left.\lim _{d \downarrow 0} N(d)(\omega)=\infty\right\}$, it follows from Theorem 3.2 that $P\{T\}=1$. Let any $\omega \in T$ and $d$ with $0<d<d_{0}$ be fixed. Using (3.4.4) in the proof of Theorem 3.4 of Isogai [6], we have

$$
\left(N h_{N}^{p}\right)^{1 / 2}\left|\sum_{m=1}^{N} a_{m} \beta_{m N} \delta_{m}\right| \leqq C_{3}\left(N h_{N}^{2 \lambda+p}\right)^{1 / 2},
$$

where $C_{3}$ is independent of $d$ and $\omega$. By (3.6.2) and (3.6.8) we get (3.6.6). Second we shall show (3.6.7). Let any $\omega \in T$ and any $d\left(0<d<d_{0}\right)$ be fixed. As $\left|f_{0}(x)-f(x)\right|$ $\leqq\|K\|_{\infty}+\|f\|_{\infty}<\infty$, it follows from (2.0.1) that 


$$
\left(N h h_{.}^{p}\right)^{1 / 2} \beta_{0 N}\left|f_{0}(x)-f(x)\right| \leqq C_{4} N^{1 / 2-a} h_{. j}^{p / 2},
$$

where $C_{4}$ is independent of $d$ and $\omega$. Combining (H1), $a>\frac{1}{2}$ and (3.6.9) we obtain (3.6.7). Finally we shall show (3.6.5). By Lemma 2.2, Lemma 3.5 and (3.6.3) we can use Theorem 1 of Anscombe [1], which implies that

$$
\left(n(d) h_{n(d)}^{p}\right)^{1 / 2} \sum_{m=1}^{N(d)} a_{m} \beta_{m N(d)} Z_{m} \underset{\mathcal{L}}{\longrightarrow} N(0, B f(x)) \quad \text { as } \quad d ! 0 .
$$

Combining (3.6.10) and Corollary 3.3, we have (3.6.5). This completes the proof. The following is one of main results.

THEOREM 3.7. Under all conditions of Theorem 3.6, we have

$$
\lim _{d \downarrow 0} P\left\{\left|f_{N(d)}(x)-f(x)\right| \leqq d\right\}=1-\alpha .
$$

ProOF. Since

$$
\begin{aligned}
& D d^{-1}\left(f_{N(d)}(x)-f(x)\right) \\
= & \left(D^{2} B f(x) /\left(N(d) h_{N(d)}^{p} d^{2}\right)\right)^{1 / 2}\left(N(d) h_{N(d)}^{p} /(B f(x))\right)^{1 / 2}\left(f_{N(d)}(x)-f(x)\right),
\end{aligned}
$$

by Theorems 3.2 and 3.6 we obtain

$$
D d^{-1}\left(f_{N(d)}(x)-f(x)\right) \underset{\mathcal{L}}{\longrightarrow} N(0,1) \quad \text { as } \quad d \downarrow 0,
$$

which yields that

$$
\begin{aligned}
& \lim _{d * 0} P\left\{\left|f_{N(d)}(x)-f(x)\right| \leqq d\right\} \\
= & P\{|N(0,1)| \leqq D\}=1-\alpha .
\end{aligned}
$$

Thus the proof is complete.

Corollary 3.8. Suppose that $f$ is loc. Lip. $\lambda$ at $x$ and $f(x)>0$. Let $\left\{h_{n}\right\}$ be given by

$$
h_{n}=n^{-r / p} \text { with } \frac{2}{3} \leqq a \leqq 1 \text { and } \frac{p}{2 \lambda+p}<r<\min \left(\frac{a p}{\lambda}, 1\right) .
$$

Then we have

$$
\lim _{d i 0} P\left\{\left|f_{N(d)}(x)-f(x)\right| \leqq d\right\}=1-\alpha .
$$

Proof. It is easily verified that the sequence $\left\{h_{n}\right\}$ given by (3.8.1) satisfies all conditions on $\left\{h_{n}\right\}$ of Theorem 3.6 with $\beta=(2 a+r-1)^{-1}$. On the other hand, by Corollary 3.3 we have $\lim _{d \downarrow 0} N(d) / n(d)=1$ with probability one, which implies (3.6.3). Thus by Theorem 3.7 we have (3.8.2).

In the remainder of this section we shall deal with the asymptotic normality of the stopping rules $N(d)$.

LEMMA 3.9. Let $\left\{\xi_{n}\right\}$ be a sequence of real numbers such that $\xi_{n}=1+o(1)$ where $o(1) \rightarrow 0$ as $n \rightarrow \infty$. Then, for any constant $b$,

$$
\xi_{n}^{b}-1=\left(\xi_{n}-1\right)\{b+o(1)\} .
$$


PROOF. By the Taylor expansion we obtain the result of lemma.

The following theorem presents the asymptotic normality.

THEOREM 3.10. Let $\left\{h_{n}\right\}$ satisfy all conditions of Theorem 3.6 replacing (H5) by the following condition:

$$
(n-1) h_{n-1}^{p} /\left(n h_{n}^{p}\right)=1+o\left(\left(n h_{n}^{p}\right)^{-3 / 2}\right) \quad \text { as } n \rightarrow \infty,
$$

where $o\left(\dot{\phi}_{n}\right)$ means that $o\left(\phi_{n}\right) / \dot{\phi}_{n} \rightarrow 0$ as $n \rightarrow \infty$. Then, under all conditions of Theorem 3.6 we have

$$
(D B)^{-1} d\left(N(d) h_{N(d)}^{p}-D^{2} B d^{-2} f(x)\right) \underset{\mathcal{L}}{\longrightarrow} N(0,1) \text { as } d ! 0 .
$$

Proof. By (H3) and (3.10.1) we get (H5). From (3.2.3) and (3.6.3) it follows that

$$
\lim _{d \downarrow 0}(N(d)-1) / n(d)=1 \text { in probability } .
$$

In the same manner as in the proof of (3.6.4) we get

$$
\left((N(d)-1) h_{N(d)-1}^{p}\right)^{1 / 2}\left(f_{N(d)-1}(x)-f(x)\right) \underset{\mathcal{L}}{\longrightarrow} N(0, B f(x))
$$

as $d \downarrow 0$. By (3.2.1) and the argument similar to Proposition 3.4 there exists a null set $\tilde{N}_{1}$ such that on $\tilde{N}_{1}^{c} N(d)<\infty$ for all d $>0, \lim _{d \downarrow 0} N(d)=\infty$ and

$$
\lim _{d \downarrow 0} f_{N(d)-1}(x)=f(x)>0 .
$$

For any $d>0$ we set $A_{d}=\left\{\omega \in \Omega: f_{N(d)-1}(x)=0\right\} \cap \tilde{N}_{1}^{c}$. By (3.10.5) we get

$$
\lim _{d \downarrow 0} P\left\{A_{d}\right\}=0 \text {. }
$$

Suppose that $N(d)<\infty$ and $f_{N(d)-1}(x)>0$. Then, by definition of $N(d)$ we have

$$
\begin{aligned}
x_{d} & \equiv\left(N(d) h_{N(d)}^{p}\right)^{1 / 2}\left(d^{2} N(d) h_{N(d)}^{p}-D^{2} B f(x)\right) \\
& \geqq D^{2} B\left(N(d) h_{N(d)}^{p}\right)^{1 / 2}\left(f_{N(d)}(x)-f(x)\right) \equiv y_{d}
\end{aligned}
$$

and

$$
\begin{aligned}
w_{d} & \equiv\left((N(d)-1) h_{N(d)-1}^{p}\right)^{1 / 2}\left(d^{2}(N(d)-1) h_{N(d)-1}^{p}-D^{2} B f(x)\right) \\
& <D^{2} B\left((N(d)-1) h_{N(d)-1}^{p}\right)^{1 / 2}\left(f_{N(d)-1}(x)-f(x)\right) \equiv v_{d} .
\end{aligned}
$$

Setting $B_{d}=\left\{\omega \in \Omega: f_{N(d)-1}(x)>0\right\} \cap \tilde{N}_{1}^{c}$, the relations (3.10.7) and (3.10.8) hold on $B_{d}$. By (H3) and (3.10.1) we get that for each $\omega \in \tilde{N}_{1}^{c}$ omitted below

$$
(N(d)-1) h_{N(d)-1}^{p} /\left(N(d) h_{N(d)}^{p}\right)=1+o(1),
$$

where $o(1) \rightarrow 0$ as $d \downarrow 0$. Let any $\omega \in \tilde{N}_{1}^{c}$ be fixed. In view of (3.10.1), (3.10.9) and Lemma 3.9 we have that as $d \downarrow 0$

$$
\left(N(d) h_{N(d)}^{p}\right)^{3 / 2}-\left((N(d)-1) h_{N(d)-1}^{p}\right)^{3 / 2}=o(1)
$$

and

$$
\left(N(d) h_{N(d)}^{p}\right)^{1 / 2}-\left((N(d)-1) h_{N(d)-1}^{p}\right)^{1 / 2}=o(1) .
$$


Hence by (3.10.10) and (3.10.11) we obtain

$$
\lim _{d \downarrow 0}\left|x_{d}-w_{d}\right|=0 \quad \text { a.s. }
$$

Let $F$ denote the distridution function of $N\left(0, D^{4} B^{3} f(x)\right)$ and let any real number $y$ be fixed. It follows from (3.10.7) that

$$
\begin{aligned}
& P\left\{x_{d} \leqq y\right\} \\
= & P\left(\left\{x_{d} \leqq y\right\} \cap B_{d}\right)-P\left(\left\{x_{d} \leqq y\right\} \cap A_{d}\right) \\
\leqq & P\left\{y_{d} \leqq y\right\}+P\left\{A_{d}\right\},
\end{aligned}
$$

where $\left\{x_{d} \leqq y\right\}$ denotes $\omega$-set such that $x_{d} \leqq y$. Since by (3.6.4) $y_{d} \underset{\mathcal{L}}{\longrightarrow} N\left(0, D^{4} B^{3} f(x)\right)$ as $d \downarrow 0$, by (3.10.6) and (3.10.13) we get

$$
\limsup _{d \downarrow 0} P\left\{x_{d} \leqq y\right\} \leqq F(y) .
$$

Let any $s>0$ be fixed. It follows (3.10.8) that for any $d>0$

which yields that

$$
\begin{aligned}
& P\left\{v_{d} \leqq y-\varepsilon\right\} \\
\leqq & P\left\{w_{d}<y-\varepsilon\right\}+P\left\{A_{d}\right\} \\
\leqq & P\left\{x_{d} \leqq y\right\}+P\left\{\left|x_{d}-w_{d}\right|>\varepsilon\right\}+P\left\{A_{d}\right\},
\end{aligned}
$$

$$
P\left\{v_{d} \leqq y-\varepsilon\right\}-P\left\{\left|x_{d}-w_{d}\right|>\varepsilon\right\}-P\left\{A_{d}\right\} \leqq P\left\{x_{d} \leqq y\right\} .
$$

Since by (3.10.4) $v_{d} \underset{\mathcal{L}}{\longrightarrow} N\left(0, D^{4} B^{3} f(x)\right)$ as $d \downarrow 0$, by (3.10.6), (3.10.12) and (3.10.15) we have $F(y-\varepsilon) \leqq \liminf _{d \downarrow 0} P\left\{x_{d} \leqq y\right\}$, which yields that as $\varepsilon \rightarrow 0$

$$
F(y) \leqq \lim _{d \downarrow 0} \inf P\left\{x_{d} \leqq y\right\} .
$$

Thus combining (3.10.14) and (3.10.16) we obtain

$$
x_{d} \underset{\mathcal{L}}{\longrightarrow} N\left(0, D^{4} B^{3} f(x)\right) \quad \text { as } \quad d \downarrow 0 .
$$

Since

$$
\begin{aligned}
& (D B)^{-1} d\left(N(d) h_{N(d)}^{p}-D^{2} B d^{-2} f(x)\right) \\
= & \left(D^{2} B f(x) /\left(N(d) h_{N(d)}^{p} d^{2}\right)\right)^{1 / 2} x_{d} /\left(D^{4} B^{3} f(x)\right)^{1 / 2},
\end{aligned}
$$

taking account of (3.2.2) and (3.10.17) we obtain (3.10.2) This completes the proof. The next lemma is a modification of Theorem (i) of Rao [7] (page 319).

LEMMA 3.11. Let $h$ be a mapping from $(0, \infty)$ into $(0, \infty)$ with $\lim _{d \rightarrow 0} h(d)=\infty$. Let $\{T(d)\}(d \in(0, \infty))$ be a family of random variables. Suppose that

$$
h(d)(T(d)-\theta) \underset{\mathcal{L}}{\longrightarrow} N\left(0, \sigma^{2}\right) \quad \text { as } \quad d \rightarrow 0,
$$

where $\theta$ is a real number and $\sigma^{2}$ is a positive number. Let $g$ be a real-valued measurable function on $R^{1}$ such that there exists the first derivative $g^{\prime}(\theta)$ of $g$ at $\theta$ with $g^{\prime}(\theta)$ 
$\neq 0$. Then

$$
h(d)(g(T(d))-g(\theta)) \underset{\mathcal{L}}{\longrightarrow} N\left(0, \sigma^{2}\left(g^{\prime}(\theta)\right)^{2}\right) \quad \text { as } \quad d \rightarrow 0 .
$$

Proof. By the Taylor expansion we get

$$
g(T(d))-g(\theta)=(T(d)-\theta)\left(g^{\prime}(\theta)+\boldsymbol{\varepsilon}(d)\right),
$$

where $\varepsilon(d) \rightarrow 0$ as $T(d)-\theta \rightarrow 0$. It follows (3.11.1) that

$$
\lim _{d \rightarrow 0} \varepsilon(d)=0 \text { in probability . }
$$

Combining (3.11.1), (3.11.3) and (3.11.4) we have (3.11.2), concluding the lemma.

COROllary 3.12. Suppose that $f$ is loc. Lip. $\lambda$ at $x$ and $f(x)>0$. Let $\left\{h_{n}\right\}$ be given by (3.8.1). Then we have

$$
(1-r) D f(x)(d \mu(d))^{-1}(N(d)-\mu(d)) \underset{\mathcal{L}}{\longrightarrow} N(0,1) \text { as } d ! 0,
$$

where $\mu(d)=\left(D^{2} B f(x) / d^{2}\right)^{1 /(1-r)}$.

PROOF. Since $r>\frac{p}{2 \lambda+p} \geqq \frac{1}{3}$ for $p \geqq 1$ and $0<\lambda \leqq 1$, we get (3.10.1). For conrenience $N$ denotes $N(d)$. As stated in Corollary 3.8 all conditions on $\left\{h_{n}\right\}$ of Theorem 3.6 are satisfied. Thus, using (3.3.1) and Theorem 3.10 we have

$$
(D B)^{-1} d\left(N^{1-r}-D^{2} B d^{-2} f(x)\right) \underset{\mathcal{L}}{\longrightarrow} N(0,1) \quad \text { as } d ! 0,
$$

which is equivalent to

$$
(d D B)^{-1}\left(d^{2} N^{1-r}-D^{2} B f(x)\right) \underset{\mathcal{L}}{\longrightarrow} N(0,1) \quad \text { as } d ! 0 .
$$

By making use of (3.12.2) and Lemma 3.11 we obtain (3.12.1), which concludes the corollary.

\section{Acknowledgement}

This paper was prepared while the author was at Kyushu University in 1979-80 on leave of absence from Niigata University. The author wishes to thank Professor N. Furukawa for his helpful suggesions and critical readings of the original manuscript.

\section{References}

[1] Anscombe, F. J., Large-sample theory of sequential estimation. Proc. Cambridge Philos. Soc., 48 (1952), 600-607.

[2] CARroll, R. J., On sequential density estimation. Z. Wahrscheinlichkeitstheorie und verw. Gebiete., 36 (1976), 137-151.

[3] Chow, Y.S. and RobBins, H., On the asymptotic theory of fixed-width sequential confidence intervals for the mean. Ann. Math. Statist., 36 (1965), 457-462.

[4] Davies, H.I. and Wegmà, E.J., Sequential nonparametric density estimation. IEEE Trans. Information Theory., IT-21 (1975), 619-628.

[5] HÁJEK, J. and RéNYI, A., Generalization of an inequality of Kolmogorov. Acta. Math. Hung., 6 (1955), 281-283. 
6. Isogar, E., Strong consistency and optimality of a sequential density estimator. Bull. Math. Statist., 19 (1980), No. 1-2, 55-69.

7- Rao, C.R., Linear statistical inference and its applications. John Wiley and Sons, N.Y., 1965.

8:- Richter, W., Limit theorems for sequences of random variables with sequences of random indeces. Theory Probability Appl., 10 (1965), 74-84.

- 9- Wegman, E.J. and DAvies, H.I., Remarks on some recursive estimators of a probability density. Ann. Statist., 7 (1979), 316-327.

10 YAMATO, H., Sequential estimation of a continuous probability density function and mode. Bull. Math. Statist., 14 (1971), No. 3-4, 1-12. 